\title{
Human Kallikrein-2, Prostate Specific Antigen and Free- Prostate Specific Antigen in Combination to Discriminate Prostate Cancer from Benign Diseases in Syrian Patients
}

\author{
Dala-Maria Bachour ${ }^{1 *}$, Emil Chahin ${ }^{2}$, Sahar Al-Fahoum²
}

\begin{abstract}
Background: The high incidence of prostate cancer as the most common malignancy in males in many countries raises the question of developing reliable detection tests. The prostate specific antigen (PSA) test is the most widely used for screening for prostate cancer; however, its low specificity elevates the number of unnecessarily biopsies. Serum human kallikrein-2 (hK2) is considered as a promising marker, and especially its ratio to fPSA, for predicting the presence of malignancy to select the best choice referring to biopsy or surveillance. In this study, we investigated the role of $\mathrm{hK} 2$ and its combinations with other markers to discriminate prostate cancer from benign diseases in Syrian patients. Materials and Methods: In this prospective oriented cross-sectional cohort study, serum samples were collected from patients referred to many Hospitals in Damascus, Syria, between May 2011 and March 2012, and diagnosed with biopsy proven benign prostate hyperplasia (BPH) or prostate cancer (PCa). Serum was analyzed for $\mathrm{hK2}$, PSA and fPSA, and the ratios of fPSA/PSA and hK2/fPSA were calculated. Results: We found that mean $\mathrm{hK2}$ /fPSA ratios were significantly higher $(\mathrm{P}=0.01)$ in prostate cancer patients than in the BPH or control groups. Also the ratio hk2/fPSA gave the largest area under the curve (AUC:0.96) which was significantly larger than for fPSA/PSA (AUC:0.41) indicative of higher specificity. Conclusions: Our results demonstrate that the ratio of hK2/fPSA might be superior to the use of fPSA/PSA alone. The hK2 could be shown to enhance the early detection of prostate cancer; especially the ratio hK2/fPSA improves specificity and hence may reduce the number of negative biopsies.
\end{abstract}

Keywords: Prostate cancer - detection tests - PSA - fPSA - hK2

Asian Pac J Cancer Prev, 16 (16), 7085-7088

\section{Introduction}

Prostate cancer is the most prevalent cancer and the second leading cause of cancer mortality among men in the western world. In Middle East countries it may be less common; but is still one of the most common cancers in men with elevated incidence especially in Lebanon and Syria (Salim et al., 2009; Shamseddin et al., 2014). Take into consideration the possibility associations between Prostate Cancer with genetic, familial, environmental and other factors, also the effect of different measures or detection programs applied in the different countries and the awareness variation of people toward these programs (Boyle et al., 2003; Arafa et al., 2012; Ezzi et al., 2014; Baade et al., 2015; Choubey et al., 2015)

The prostate cancer had few specific symptoms in its early stage, for that reason, detecting cancer holds several difficult. The standard practice of screening for prostate cancer includes PSA, with or without digital rectal exam test (DRE), starting at the age of 50 years in all males or earlier in men with certain risk factors. However, only according to the prostate biopsy results we can confirm the prostate cancer diagnosis (Shariat et al., 2008; Madu et al., 2010; Heidenreich et al., 2014).

PSA levels influenced by many factors like age, race, the Metabolic Syndrome or even Body Mass Index (BMI) and alcohol consumption (Chiu et al., 2011; Bashir et al., 2014; Daniyal et al., 2014; Ozbek et al., 2014; Woo et al., 2014). But the most important disadvantage of the PSA test is its low specificity which means that a high percentage of the men are negative on initial biopsy (Jansen et al., 2009). This lack of specificity of PSA warrants the use of new biomarkers to diagnose prostate cancer more accurately and decreases the number of unnecessary biopsies (Madu et al., 2010; Murray et al., 2014; Esfahani et al., 2015).

Kallikrein-related peptidases represent a subgroup of the serine protease family consisting of 15 members located at q13.4 of chromosome 19. Of the members of the kallikrein family, hK2 has a valuable importance since its levels are significantly higher in men with prostate

${ }^{1}$ Master Student, Faculty of Pharmacy, ${ }^{2}$ Department of Clinical Biochemistry and Microbiology, Damascus University, Syria *For correspondence: dalaba33@yahoo.com 


\section{Dala-Maria Bachour et al}

cancer compared with men with benign diseases. HK2 is a $28 \mathrm{kDa}$ serine protease produced by prostatic epithelial cells. Structurally, hK2 and PSA (hK3) share the highest sequence homology (in KLKS family). The serum level of hK2 increases in many conditions including leaking from the disrupted prostatic cells and over expression in cancerous prostate tissue which increases with increasing grade (Herrala et al., 2001; Borgoño et al., 2004; Jansen et al., 2009).

Therefore, many studies indicate that measurement of serum hK2 with PSA can improve diagnosis of prostate cancer, importantly, the ratio of hK2/fPSA ratio alone or combined with fPSA/PSA ratio enhances the discrimination between prostate cancer and BPH and may also be useful in grading the cancer (Becker et al., 2000; Stephan et al., 2006; Raaijmakers et al., 2007). All these advantages suggest that a panel or multiple markers including $\mathrm{hK} 2$ is an important biomarker for the presence and recurrence of cancer (Martin et al., 2004; Vickers et al., 2008; Thorek et al., 2013).

In this study, we investigate the role of hK2 and its combinations with other markers to discriminate prostate cancer from benign diseases in Syrian patients.

\section{Materials and Methods}

\section{Patient selection}

Included in this prospective oriented cross-sectional cohort study, were 70 patients who were referred to either the Urology department in Al-Bairouni university Hospital or Al-Mouwasat university Hospital or Ibn Al-Nafees Hospital in Damascus, Syria. Between May 2011 and March 2012 and aged between 45 - 83 years old. These patients underwent prostatic TRUS guided needle-core prostatic biopsies because of either PSA values of 4.0 $\mathrm{ng} / \mathrm{mL}$ or abnormal digital rectal examination (DRE), later they were diagnosed with either PCa (35 patients) or BPH (35 patients) according to the histological tests results and Grade was evaluated by the Gleason scoring system according to it patients classified into three groups: low-grade group (GS=2-3-4), intermediate-grade group (GS=5-6-7) and high-grade group (GS=8-9-10). Patients present with previous cancer, previous prostate biopsy or metastatic diseases were excluded.

Blood samples were collected before DRE and biopsy procedure and any treatment. All participants agreed to provide blood samples and had signed a written informed consent; the study has been approved by the ethics committee.

Plasma was separated from blood samples and was stored at $-70^{\circ} \mathrm{C}$. PSA, fPSA and hK2 were analyzed at a later time in the frozen serum samples. Both fPSA and PSA levels were analyzed with commercially ELISA kits (R\&D Systems) and hK2 levels were analyzed using a for-research only ELISA kit (Uscn Life Science Inc.), according to the manufacturer's recommendations.

\section{Statistical analysis}

Statistical analyses were done with commercially available software using SPSS program version 19 and
Microsoft Excel 2007. The analyses of differences between these variables in the three groups were performed with the T-student test for normally distributed parameters. Relationships between different variables were assessed by the Spearman correlation coefficient. For all analyses, $\mathrm{P}<0.05$ was considered statistically significant. ROC curves were constructed for PSA, fPSA, the fPSA/PSA ratio, hK2 and hK2/fPSA ratio, plotting sensitivity vs. (1-specificity), and the areas under the ROC curves (AUCs) were calculated.

\section{Results}

All 70 patients underwent prostatic TRUS guided needle-core prostatic biopsy, divided according the histological tests results into a cancer group (35 patients) and a BPH group (35 patients). PSA, fPSA and hK2 were measured and the fPSA/PSA and the hK2/fPSA ratios were calculated, the results are presented in Table I.

The range of age values from 47 to 83 years in $\mathrm{PCa}$ patients, with a mean \pm SE of $78 \pm 5.3$ years and from 45 to 82 years in BPH patients, with the mean \pm SE value being $76 \pm 6.1$ years. Values did not reach statistical significance between the $\mathrm{PCa}$ and $\mathrm{BPH}$ groups $(\mathrm{P}=0.12)$. The range of PSA values from 3.71 to $146.3 \mathrm{ng} / \mathrm{ml}$ in PCa patients, with a mean \pm SE of $21.1 \pm 13.8 \mathrm{ng} / \mathrm{ml}$ and from 1.69 to $41.2 \mathrm{ng} / \mathrm{ml}$ in $\mathrm{BPH}$ patients, with the mean $\pm \mathrm{SE}$ value being $12.3 \pm 3.97 \mathrm{ng} / \mathrm{ml}$. Values did not reach statistical significance between the $\mathrm{PCa}$ and $\mathrm{BPH}$ groups $(\mathrm{P}=0.11)$. fPSA/PSA ratio values ranged from 0.69 to $47.5 \%$ in PCa patients, with a mean \pm SE of $13.4 \pm 5.13 \%$ and from 2.55 to $72.3 \%$ in $\mathrm{BPH}$ patients, with the mean $\pm \mathrm{SE}$ value being $25.1 \pm 7.53 \%$. Values also did not reach statistical significance between the $\mathrm{PCa}$ and $\mathrm{BPH}$ groups $(\mathrm{P}=0.06)$. hK2/fPSA ratio values ranged from 6.11 to $539.8 \%$ in PCa patients, with a mean \pm SE of $156.6 \pm 60.1 \%$ and from 1.39 to $42.7 \%$ in $\mathrm{BPH}$ patients, with the mean \pm SE value being $51.9 \pm 18.6 \%$. Values were significantly higher in $\mathrm{PCa}$ than BPH $(\mathrm{P}=0.01)$.

The Gleason score (GS) values ranged from 3 to 9 in 35 patients diagnosed of cancer, with a mean 6.8. Of them $8(22.9 \%)$ patients classified in low-grade group, 16 $(45.7 \%)$ patients classified in intermediate-grade group and $11(31.4 \%)$ patients classified in high-grade group.

In general, the levels of PSA, fPSA/PSA ratio and hK2/fPSA ratio were higher in cancer patients; however, the differences in fPSA/PSA ratios values did not reach

Table 1. Mean Values and Ranges of the Variables

\begin{tabular}{lccc}
\hline & $\begin{array}{c}\text { PCa }(\mathrm{n}=35) \\
\text { Mean (range) }\end{array}$ & $\begin{array}{c}\text { BPH }(\mathrm{n}=35) \\
\text { Mean (range) }\end{array}$ & P value \\
\hline Age, years & 78 & 76 & 0.12 \\
& $(47-83)$ & $(45-82)$ & \\
PSA, ng/ml & 21.1 & 12.3 & 0.11 \\
fPSA/PSA ratio, \% & $13.71-146.3)$ & $(1.69-41.2)$ & \\
& $(0.69-47.5)$ & 25.1 & 0.06 \\
hK2/fPSA ratio, \% & 156.6 & 51.9 & $0.01 *$ \\
& $(6.11-539.8)$ & $(1.39-42.7)$ & \\
\hline
\end{tabular}

*PSA: Prostate Specific Antigen, fPSA: free Prostate Specific Antigen, PSA: Prostate Specific Antigen, hK2: Human Kallikrein-2, BPH: benign prostate hyperplasia, PCa: prostate cancer; * Significant 


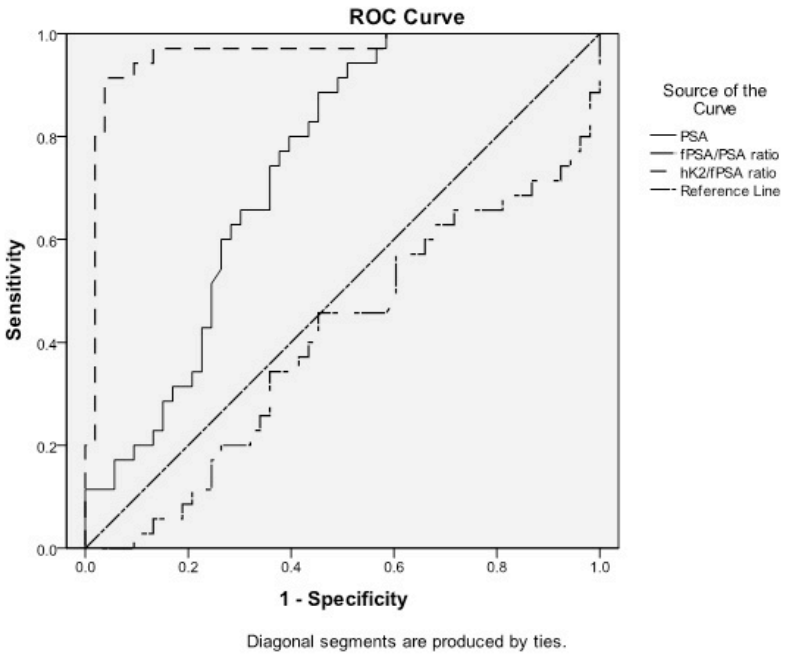

Figure 1. ROC analyses for PSA, fPSA/PSA ratio and hK2/fPSA ratio

statistical significance between the $\mathrm{PCa}$ and $\mathrm{BPH}$ groups $(\mathrm{P}=0.06)$. Values were significantly higher in $\mathrm{PCa}$ than $\mathrm{BPH}(\mathrm{P}=0.01)$ for $\mathrm{hK} 2 / \mathrm{fPSA}$ ratio in this study sample.

ROC analyses were performed to show the relative potential of the PSA, fPSA/PSA ratio and hK2/fPSA ratio for discriminating prostate cancer from benign prostatic hyperplasia are: PSA (AUC:0.74, 95\% CI, 0.64-0.84), fPSA/PSA ratio (AUC:0.41, 95\% CI, 0.29-0.53), hK2/ fPSA (AUC:0.96, 95\% CI, 0.92-1,0) presented in Figure 1 ; which assure that diagnostic characteristics of kh2/fPSA ratio is better and gives the best discriminatory power of the other tests.

\section{Discussion}

More studies suggest that there is no single marker could be used for the detection of $\mathrm{PCa}$, and the combination of multiple markers improves the specificity of detecting PCa and makes it statistically stronger, in order to achieve best $\mathrm{PCa}$ detection by reducing the number of the unnecessary biopsies.

Previous studies have indicated that $\mathrm{hK} 2$ has a valuable importance since its levels are significantly higher in men with prostate cancer; based in on these findings and considering many factors like the aging of the population, reporting delay and ethnicity, we decided to investigate the $\mathrm{hK} 2$ role and its combinations with PSA and PPSA in the detection of PCa program applied in some Syrian hospitals; witch include PSA tests with or without DRE examination. Our results were compatible with the previous studies as we found that hK2, fPSA, and PSA combinations are valuable in predicting $\mathrm{PCa}$ in our population.

As shown in Table I, PSA levels or fPSA/PSA ratio did not differ significantly between the two groups $(\mathrm{P}=0.11$ and $\mathrm{P}=0.06$ respectively). However the ratio $\mathrm{hK} 2 / \mathrm{fPSA}$ did distinguish significantly between $\mathrm{PCa}$ and $\mathrm{BPH}$ patients $(\mathrm{P}=0.01)$.

Our findings are similar to the Scorilas et al. study which reported that some kallikreins are elevated in many malignancies, especially hK2 which increase in prostate cancer so it may be used as a marker in this cancer (Scorilas et al., 2003). Subsequently, Nam et al. and Martin et al studies demonstrate the utility of the ratio hK2/fPSA to discriminate prostate cancer patients from non-cancer men ( $\mathrm{P}>0.0001)$, suggesting that this ratio may have a useful diagnostic role (Nam et al., 2000; Martin et al., 2004).

We also found that the ROC curves show a good diagnostic performance by high AUCs for both PSA levels and PPSA/PSA ratio alone (AUC:0.74 and AUC:0.41 respectively), but a much stronger effect for the hK2/ fPSA ratio (AUC:0.96).

These findings agrees with Raaijmakers et al. which confirm that the ratio hK2/fPSA was a better diagnosis marker than the ratio fPSA/PSA alone according to the ROC curves analysis and results (Raaijmakers et al., 2007). Also Martin et al. results who approves that hK2/ fPSA ratio (AUC: 08) was useful in discriminating the two diseases (PCa and BPH) (Martin et al., 2004). Similarly, Becker et al. and Scorilas et al. studies showed a higher specificity of hK2/fPSA ratio vs. fPSA/PSA ratio in low PSA levels (Becker et al., 2000; Scorilas et al., 2003).

An explanation of this effect may be found in the position of fPSA as denominator and hK2 as numerator. Because the levels of fPSA are lower in men with PCa and the levels of $\mathrm{hK} 2$ is higher in men with $\mathrm{PCa}$, the ratio could result in a test with more discriminating power.

Hence, our results showed an improved specificity of PCa detection when combined hK2 with PSA and PPSA.

We need to mention that unlike PSA test, the DRE exam was not performed to all patients. Hence, we notice that most of the patients (16/35) were classified in intermediate-grade group, with only $8 / 35$ patients in low-grade group.

Several limitations of our study deserve mentioning. The small number of patients included in our study mainly caused by financial limitations was an obstacle especially in having a more reliable statistical analysis data.

Also, the levels of fPSA may be influenced by freezing our samples at $-80^{\circ} \mathrm{C}$; although the freezing was as soon as possible after centrifuging and the analysis was made immediately after thawing in order to avoid fPSA degradation in vitro.

As a conclusion, our results demonstrate that the measurement of serum hK2 levels is very important in prostate cancer detection because we have found that the use of ratio of hK2/fPSA is better than the use of fPSA/ PSA alone since hK2/fPSA offered the most statistical power in detecting prostate cancer, as measured by the AUC on ROC analysis.

We believe our study should be looked upon as a demonstration of the potential of $\mathrm{hK} 2$ assisting to predict the biopsy results for PCa. The hK2 has been shown to add important information in the detection of prostate cancer; especially the ratio hK2/fPSA improves specificity and hence reduces the number of unnecessary biopsies.

\section{References}

Arafa MA, Rabah DM, Wahdan IH (2012). Awareness of general public towards cancer prostate and screening practice in 
Dala-Maria Bachour et al arabic communities: a comparative multi-center study. Asian Pac J Cancer Prev, 13, 4321-6.

Baade PD, Yu XQ, Smith DP, et al (2015). Geographic disparities in prostate cancer outcomes--review of international patterns. Asian Pac J Cancer Prev, 16, 1259-75.

Bashir MN,Ahmad MR, Malik A (2014). Risk factors of prostate cancer: a case-control study in faisalabad, pakistan. Asian Pac J Cancer Prev, 15, 10237-40.

Becker C, Piironen T, Pettersson K, et al (2000). Clinical value of human glandular kallikrein 2 and free and total prostatespecific antigen in serum from a population of men with prostate-specific antigen levels $3.0 \mathrm{ng} / \mathrm{mL}$ or greater. Urol, $\mathbf{5 5}, 694-9$.

Borgoño CA, Diamandis EP (2004). The emerging roles of human tissue kallikreins in cancer. Nat Rev Cancer, $\mathbf{4}$, 876-90.

Boyle P, Severi G, Giles GG. (2003). The epidemiology of prostate cancer. Urol Clin North Am, 30, 209-17.

Chiu PK, Wong AY, Hou SM, et al (2011). Effect of body mass index on serum prostate-specific antigen levels among patients presenting with lower urinary tract symptoms. Asian Pac J Cancer Prev, 12, 1937-40.

Choubey VK, Sankhwar SN, Carlus SJ, et al (2015). SRD5A2 gene polymorphisms and the risk of benign prostatic hyperplasia but not prostate cancer. Asian Pac J Cancer Prev, 16, 1033-6.

Daniyal M, Siddiqui ZA, Akram M, et al (2014). Epidemiology, etiology, diagnosis and treatment of prostate cancer. Asian Pac J Cancer Prev, 15, 9575-8.

El Ezzi AA, Zaidan WR, El-Saidi MA, et al (2014). Association of benign prostate hyperplasia with polymorphisms in $\mathrm{vdr}$, cyp17, and srd5a2 genes among lebanese men. Asian Pac J Cancer Prev, 15, 1255-62.

Esfahani M, Ataei N, Panjehpour M (2015). Biomarkers for evaluation of prostate cancer prognosis. Asian Pac J Cancer Prev, 16, 2601-11.

Heidenreich A, Bellmunt J, Bolla M, et al (2011). EAU guidelines on prostate cancer. part 1: screening, diagnosis, and treatment of clinically localized disease. Europ Urol, 59, 61-71.

Herrala AM, Porvari KS, Kyllonen AP, et al (2001). Comparison of human prostate specific glandular kallikrein 2 and prostate specific antigen gene expression in prostate with gene amplification and overexpression of prostate specific glandular kallikrein 2 in tumor tissue. Cancer, 92, 2975-84.

Jansen FH, Roobol M, Jenster G, et al (2009). Screening for prostate cancer in 2008 II: the importance of molecular subforms of prostate-specific antigen and tissue kallikreins. European Urol, 55, 563-74.

Madu CO, Lu Y (2010). Novel diagnostic biomarkers for prostate cancer. J Cancer, 6, 150-77.

Martin BJ, Finlay JA, Sterling K, et al (2004). Early detection of prostate cancer in african-american men through use of multiple biomarkers: human kallikrein 2 (hK2), prostatespecific antigen (PSA), and free PSA (fPSA). Prostate Cancer Prostatic Diseases, 7, 132-7.

Murray NP, Reyes E, Fuentealba C, (2014). Extended use of p504s positive primary circulating prostate cell detection to determine the need for initial prostate biopsy in a prostate cancer screening program in chile. Asian Pac J Cancer Prev, 15, 9335-9.

Nam RK, Diamandis EP, Toi A, et al (2000). Serum human glandular kallikrein-2 protease levels predict the presence of prostate cancer among men with elevated prostate-specific antigen. J Clin Oncol, 18, 1036-42.

Ozbek E, Otunctemur A, Dursun M,(2014). The metabolic syndrome is associated with more aggressive prostate cancer.
Asian Pac J Cancer Prev, 15, 4029-32.

Raaijmakers R, de Vries SH, Blijenberg BG, et al (2007). $\mathrm{hK} 2$ and free PSA, a prognostic combination in predicting minimal prostate cancer in screen-detected men within the PSA range 4-10 ng/ml. Eur Urol, 52, 1358-64.

Salim EI, Moore MA, Al-Lawati JA, et al (2009). Cancer epidemiology and control in the arab world - past, present and future. Asian Pac J Cancer Prev, 10, 3-16.

Scorilas A, Plebani M, Mazza S, et al (2003). Serum human glandular kallikrein (hK2) and insulin likegrowth factor1 (IGF-1) improve the discrimination between prostate cancer and benign prostatic hyperplasia in combination with total and \% free PSA. Prostate, 54, 220-9.

Shamseddine A, Saleh A, Charafeddine M, et al (2014). Cancer trends in Lebanon: a review of incidence rates for the period of 2003-2008 and projections until 2018. Popul Health Metr, 12, 4 .

Shariat SF, Roehrborn CG (2008). Using biopsy to detect prostate cancer. Rev Urol, 10, 262-80.

Stephan C, Jung K, Nakamura T, et al (2006). Serum human glandular kallikrein $2(\mathrm{hK} 2)$ for distinguishing stage and grade of prostate cancer. Int J Urol, 13, 238-43.

Thorek DL, Evans MJ, Carlsson SV, et al (2013). Prostatespecific kallikrein-related peptidases and their relation to prostate cancer biology and detection. Established relevance and emerging roles. Thromb Haemost, 110, 484-92.

Vickers AJ, Cronin AM, Aus G, Pihl CG, et al (2008). A panel of kallikrein markers can reduce unnecessary biopsy for prostate cancer: data from the european randomized study of prostate cancer screening in Goteborg, Sweden. BMC Med, $6,19$.

Woo HY, Park H, Kwon MJ, et al (2012). Association of prostate specific antigen concentration with lifestyle characteristics in Korean men. Asian Pac J Cancer Prev, 13, 5695-9. 